Vol. 41 (1990) [201-206]

\title{
ON COMMUTATIVITY OF RINGS WITH SOME POLYNOMIAL CONSTRAINTS
}

\author{
Mohd. Ashraf and Murtaza A. Quadri
}

Let $R$ be an associative ring with unity $1, N(R)$ the set of nilpotents, $J(R)$ the Jacobson radical of $R$ and $n>1$ be a fixed integer. We prove that $R$ is commutative if and only if it satisfies $(x y)^{n}=y^{n} x^{n}$ for all $x, y \in R \backslash N(R)$ and commutators in $R$ are $n(n+1)$-torsion free. Moreover, we extend the same result in the case when $x, y \in R \backslash J(R)$.

\section{INTRODUCTION}

Throughout $R$ will denote an associative ring with unity 1 . Let us denote the centre of ring $R$ by $Z(R)$, the commutator ideal by $C(R)$, the Jacobson radical by $J(R)$, the set of nilpotents by $N(R)$ and for any $x, y$ in $R,[x, y]=x y-y x$. Let $n>1$ be a fixed integer. We consider the following properties:

(I) $(x y)^{n}=x^{n} y^{n}$, for all $x, y$ in $R$.

(II) $(x y)^{n}=x^{n} y^{n}$, for all $x, y$ in $R \backslash N(R)$.

(III) $(x y)^{n}=x^{n} y^{n}$, for all $x, y$ in $R \backslash J(R)$.

(IV) $(x y)^{n}=y^{n} x^{n}$, for all $x, y$ in $R$.

(V) $(x y)^{n}=y^{n} x^{n}$, for all $x, y$ in $R \backslash N(R)$.

(VI) $(x y)^{n}=y^{n} x^{n}$, for all $x, y$ in $R \backslash J(R)$.

(VII) For all $x, y$ in $R n(n+1)[x, y]=0$ implies $[x, y]=0$.

A well-known theorem of Herstein [6] asserting that a ring $R$, which satisfies (I), must have nil commutator ideal, has recently been generalised by Bell [4] as follows: "If $R$ is an $n$-torsion free ring and satisfies (I) for two consecutive integers $n, n+1$, then $R$ is commutative". Abu-Khuzam [1] proved that if $R$ is an $n(n-1)$-torsion free ring satisfying (I), then $R$ is commutative. Further, Bell and Yaqub [5] established commutativity of the ring $R$ for the case when condition (I) is replaced by either of conditions (II) or (III) in the hypotheses of the last result. In [11] the authors proved that if $R$ satisfies (IV) for two consecutive integers $n, n+1$ together with the condition $(x+y)^{n}=x^{n}+y^{n}$, then $R$ is commutative. In this direction we prove the following:

THEOREM 1. A ring $R$ is commutative if and only if it satisfies (V) and (VII).

THEOREM 2. A ring $R$ is commutative if and only if it satisfies (VI) and (VII).

Received 7 March 1989

Copyright Clearance Centre, Inc. Serial-fee code: 0004-9729/90 \$A2.00+0.00. 


\section{MAIN RESULTS}

The following results are pertinent for developing the proofs of the above theorems. Proofs of Lemma 1 and Lemma 2 can be found in [3] and [8] respectively. Moreover, they hold even for rings without unity. Lemma 3 has also been proved in [9], but here we are supplying an independent and elementary proof.

Lemma 1. Let $R$ be a ring satisfying an identity $q(X)=0$, where $q(X)$ is a polynomial in noncommuting indeterminates, its coefficients being integers with highest common factor one. If there exists no prime $p$ for which the ring of $2 \times 2$ matrices over $G F(p)$ satisfies $q(X)=0$, then $R$ has a nil commutator ideal and the nilpotent elements of $R$ form an ideal

Lemma 2. If $x, y \in R$ and $[x,[x, y]]=0$, then $\left[x^{m}, y\right]=m x^{m-1}[x, y]$ for all positive integers $m$.

Lemma 3. Let $R$ be a ring and $f: R \rightarrow R$ be a function such that $f(x+1)=$ $f(x)$ holds for all $x$ in $R$. If for some positive integer $m, x^{m} f(x)=0$ for all $x$ in $R$, then necessarily $f(x)=0$.

Proof: We have $f(x)=\{(1+x)-x\}^{2 m+1} f(x)$. On expanding the right hand side expression by the binomial theorem and using the fact that $x^{m} f(x)=0$ and $(1+x)^{m} f(x)=0$, we immediately get $f(x)=0$.

Proof of TheOREM 1: Let $u, v$ be units in $R$. On replacing $x$ by $u^{-1} v$ and $y$ by $u$ in the given condition, we get

$$
u^{-1} v^{n} u=\left(u^{-1} v u\right)^{n}=u^{n}\left(u^{-1} v\right)^{n}=u^{n} v^{n} u^{-n}
$$

which implies that

$$
\left[u^{n+1}, v^{n}\right]=0 \text { for all units } u, v \text { in } R \text {. }
$$

This readily yields that $\left[u^{n+1},\left(v^{n}\right)^{n+1}\right]=0,\left[u^{n},\left(v^{n+1}\right)^{n}\right]=0$ and hence

$$
\left[u, v^{n(n+1)}\right]=0 \text { for all units } u, v \text { in } R \text {. }
$$

Let $a \in N(R)$. Then there exists a minimal positive integer $p$ such that

$$
\left[u, a^{k}\right]=0 \text { for all integers } k \geqslant p .
$$

Suppose $p>1$, then $\left(1+a^{p-1}\right)$ is a unit in $R$ and hence by (2) and (3) we have, $0=\left[u,\left(1+a^{p-1}\right)^{n(n+1)}\right]=n(n+1)\left[u, a^{p-1}\right]$, which implies that $\left[u, a^{p-1}\right]=0$. This contradicts the minimality of $p$. Hence $p=1$ and (3) implies that

$$
[u, a]=0 \text { for all units } u \text { in } R \text { and all nilpotents } a \text { in } R \text {. }
$$


Now suppose $x \in R$ and again $u$ is a unit in $R$. If $u x \in N(R)$, then by (4), $[u, u x]=0$ and hence $[u, x]=0$. Next, suppose that $u x \notin N(R)$, then $u x^{n} u^{-1}=\left((u x) u^{-1}\right)^{n}=$ $u^{-n} x^{n} u^{n}$ and hence $\left[u^{n+1}, x^{n}\right]=0$. Using the same arguments as used to get (4) from (2), we have

$$
\left[a, x^{n}\right]=0 \text { for all } x \text { in } R \text { and all nilpotents } a \text { in } R \text {. }
$$

Let $S=\left\langle x^{n}: x \in R\right\rangle$ be the subring generated by all $n$th powers of elements of $R$. Thus (5) implies that $N(S) \subseteq Z(S)$. Clearly $S$ satisfies the hypotheses placed on $R$. In fact, all nilpotent elements of $S$ are central and hence $S$ satisfies $(x y)^{n}=y^{n} x^{n}$ for all $x, y$ in $S$, which is a polynomial identity with coprime integral coefficients. But if we consider $x=e_{12}, y=e_{21}$, we find that no $2 \times 2$ matrices over $G F(p), p$ a prime satisfies this identity. Hence by Lemma 1, the commutator ideal $C(S)$ of $S$ is nil. Thus in view of the above arguments we get

$$
C(S) \subseteq N(S) \subseteq Z(S) .
$$

For all $x, y$ in $S,(x y)^{n} x=x(y x)^{n}$, which implies that $\left[x^{n+1}, y^{n}\right]=0$.

Now using (6) and Lemma 2, we have $(n+1) x^{n}\left[x, y^{n}\right]=0$ and hence $x^{n}\left[x,(n+1) y^{n}\right]=0$. Replacing $x$ by $(x+1)$ we see that $(x+1)^{n}\left[x,(n+1) y^{n}\right]=0$, and hence, by Lemma $3,(n+1)\left[x, y^{n}\right]=0$. Using hypothesis (VII), we get $\left[x, y^{n}\right]=0$. Again using (6), Lemma 2, Lemma 3 and hypothesis (VIII), we conclude that $[x, y]=0$ for all $x, y$ in $S$. Therefore

$$
\left[x^{n}, y^{n}\right]=0 \text { for all } x, y \text { in } R .
$$

Now we observe that $x^{n+1} y^{n+1}=x\left(x^{n} y^{n}\right) y=x(y x)^{n} y$, that is

$$
x^{n+1} y^{n+1}=(x y)^{n+1} \text { for all } x, y \text { in } R \backslash N(R) \text {. }
$$

If $u, v$ are units in $R$, then we find that

$$
\left[u^{n+1}, v^{n}\right]=0 \text { for all units } u, v \text { in } R .
$$

Similar arguments to those used in getting (5) from (1) yield the following from (6) (use $(8)$ in place of $(V))$.

$$
\left[a, x^{n+1}\right]=0 \text { for all } x \text { in } R \text { and all nilpotents } a \text { in } R .
$$

Thus (5) and (10) yield, $[a, x]=0$ for all $x$ in $R$ that is $N(R) \subseteq Z(R)$, But by [7], (7) yields that the commutator ideal $C(R)$ of $R$ is nil. Hence we have

$$
C(R) \subseteq N(R) \subseteq Z(R) .
$$


To complete the proof of our theorem, let $x, y \in R$. By (7), $\left[x^{n}, y^{n}\right]=0$. Using (11) and Lemma 2, we find that $n x^{n-1}\left[x, y^{n}\right]=0$. Replace $x$ by $(x+1)$ to get $(x+1)^{n}\left[x, n y^{n}\right]=0$. Hence by Lemma $3, n\left[x, y^{n}\right]=0$ and using hypothesis (VII) we get $\left[x, y^{n}\right]=0$. Applying the same argument to $\left[x, y^{n}\right]=0$, we see that $y^{n-1}[x, n y]=$ $0=(y+1)^{n-1}[x, n y]$ and hence by Lemma 3 and (VII), $[x, y]=0$. This completes the proof.

As a consequence of the above theroem, we can derive the following:

CoROLlary . Let $R$ be a ring satisfying (IV) and (VII). Then $R$ is commutative.

Proof of Theorem 2: The arguments used in the proof of Theorem 1 are still valid in the present situation. Hence, we get

$$
\begin{aligned}
& {\left[u, v^{n(n+1)}\right]=0 \text { for all units } u, v \text { in } R .} \\
& {[u, a]=0 \text { for all } a \in N(R) \text { and all units } u \text { in } R}
\end{aligned}
$$

Now if $x, y \in J(R)$, then (1) yields

$$
\left[(1+x),(1+y)^{n(n+1)}\right]=0 \text { for all } x, y \text { in } J(R)
$$

Using the structure theory of rings, it can easily be verified that if $R$ is a semisimple ring satisfying $(x y)^{n}=y^{n} x^{n}$, then $R$ is commutative. Thus by (VI), $R / J(R)$ is commutative and hence

$$
C(R) \subseteq J(R)
$$

Now if $c_{1}=\left[x_{1}, y_{1}\right], c_{2}=\left[x_{2}, y_{2}\right], c_{3}=\left[x_{3}, y_{3}\right]$ be any commutators then by $(3)$ and (4), we get

$$
\left[\left(1+c_{3}\right),\left(1+c_{1}+c_{2}+c_{1} c_{2}\right)^{n(n+1)}\right]=0
$$

This is a polynomial identity satisfied by all elements of $R$. Now consideration of $c_{1}=c_{3}=\left[e_{11}, e_{11}+e_{12}\right], c_{2}=\left[e_{11}+e_{12}, e_{21}\right]$ shows that no $2 \times 2$ matrices over $G F(p), p$ a prime, satisfies (5) and hence by Lemma 1 , the commutator ideal of $R$ is nil and the set of nilpotents form an ideal. Combining this with (2), we find that

$$
[(1+x),[(1+x),(1+y)]]=0 \text { for all } x, y \in J(R)
$$

Using (3), (6) and Lemma 2, we get $n(n+1)(1+y)^{n^{2}+n-1}[1+x, 1+y]=0$ that is $(1+y)^{n^{2}+n-1}[1+x, n(n+1)(1+y)]=0$. Hence by (VII), [x,y]=0 for all $x, y$ in 
$J(R)$. Thus $J(R)$ is commutative and $(J(R))^{2}$ is central. An easy induction shows that

$$
(x y)^{k}=y^{k} x^{k} \text { for all } k \geqslant 2 ; x, y \in J(R) .
$$

Combining (7) together with (VI), we find that $R$ satisfies (IV). Hence the commutativity of $R$ follows by the Corollary to Theorem 1 .

The noncommutative ring of $3 \times 3$ strictly upper triangular matrices over the ring $Z$ of integers shows that the existence of unity 1 in both the theorems is essential. Further, we provide the following example to show that condition (VII), where commutators are $n(n+1)$-torsion free cannot be replaced by " $n$-torsion free" or " $(n+1)$-torsion free" even if the given condition $(x y)^{n}=y^{n} x^{n}$ holds for all $x, y$ in $R$.

EXAMPLE. Let

$$
R=\left\{a I+B \mid B=\left(\begin{array}{ccc}
0 & b & c \\
0 & o & d \\
0 & o & o
\end{array}\right), I=\left(\begin{array}{ccc}
1 & o & o \\
0 & 1 & 0 \\
0 & o & 1
\end{array}\right), a, b, c, d \in G F(3)\right\}
$$

It can easily be verified that $(x y)^{2}=y^{2} x^{2}$ and $(x y)^{3}=y^{3} x^{3}$. Thus with $n=2$, $n[x, y]=0$ implies $[x, y]=0$ and $(x y)^{n}=y^{n} x^{n}$. Also with $n=3,(n+1)[x, y]=0$ implies that $[x, y]=0$ and $(x y)^{n}=y^{n} x^{n}$ for all $x, y$ in $R$. However, $R$ is not commutative.

\section{REFERENCES}

[1] Hazar Abu-Khuzam, 'A commutativity theorem for rings', Math. Japonica 25 (1980), 593-595.

[2] Hazar Abu-Khuzam, 'Commutativity results for rings', Bull. Austral. Math. Soc. 38 (1988), 191-195.

[3] H.E. Bell, 'On some commutativity theorem of Herstein', Arch. Math. 24 (1973), 34-38.

[4] H.E. Bell, 'On the power map and ring commutativity', Canad. Math. Bull. 21 (1978), 399-404.

[5] H.E. Bell and A. Yaqub, 'Commutativity of rings with certain polynomial constraints', Math. Japonica 32 (1987), 511-519.

[6] I.N. Herstein, 'Power maps in rings', Michigan Math. J. 8 (1961), 29-32.

[7] I.N. Herstein, 'A commutativity theorem', J. Algebra 38 (1976), 112-118.

[8] N. Jacobson, Structure of rings (Amer. Math. Soc. Colloq. Publ. 37, 1964).

[0] W.K. Nicholson and A. Yaqub, 'A commutativity theorem for rings and groups', Canad. Math. Bull. 22 (1979), 419-423.

[10] M.A. Quadri and M. Ashraf, 'A theorem on commutativity of semi prime rings', Bull. Austral. Math. Soc. 34 (1986), 411-413. 
[11] M.A. Quadri and M. Ashraf, 'A remark on a commutativity condition for rings', (submitted).

Department of Mathematics

Aligarh Muslim University

Aligarh 202002

India 